\title{
Spurious source generation in mapping from noisy phase-self-calibrated data
}

\author{
I. Martí-Vidal and J. M. Marcaide \\ Dpt. Astronomia i Astrofísica, Universitat de València, C/Dr. Moliner 50, 46100 Burjassot (Valencia), Spain \\ e-mail: I.Marti-Vidal@uv.es
}

Received 17 September 2007 / Accepted 12 December 2007

\begin{abstract}
Phase self-calibration (or selfcal) is an algorithm often used in the calibration of interferometric observations in astronomy. Although a powerful tool, this algorithm presents strong limitations when applied to data with a low signal-to-noise ratio. We analyze the artifacts that the phase selfcal algorithm produces when applied to extremely noisy data. We show how the phase selfcal may generate a spurious source in the sky from a distribution of completely random visibilities. This spurious source is indistinguishable from a real one. We numerically and analytically compute the relationship between the maximum spurious flux density generated by selfcal from noise and the particulars of the interferometric observations. Finally, we present two simple tests that can be applied to interferometric data for checking whether a source detection is real or whether the source is an artifact of the phase self-calibration algorithm.
\end{abstract}

Key words. techniques: interferometric - methods: data analysis - techniques: image processing

\section{Introduction}

Phase self-calibration (or selfcal) is an algorithm often used in the calibration of radio astronomical data. It was introduced by Readhead \& Wilkinson (1978) and Cotton (1979), and it has been essential for the success of Very Long Baseline Interferometry (VLBI) imaging. Also, the antennabased calibrations obtained from the Global Fringe Fitting algorithm (Schwab \& Cotton 1983) are equivalent to a phase self-calibration. The phase selfcal will also be an algorithm widely used with future interferometric instruments, such as the Atacama Large Millimeter Array (ALMA) or the Square Kilometre Array (SKA), now under construction or planned. Optical interferometric observations (like those in the Very Large Telescope Interferometry, VLTI) will also eventually benefit from some form of selfcal, although closure phases and amplitudes are measured in optical interferometry in a very different way than in radio. Thus, the statistical analysis presented here may need some substantial changes to rigorously describe the probability of false detections by optical interferometers.

Given that part of the interferometric observations obtained from all those instruments may come from very faint sources, it is important to take into account the undesired and uncontrollable effects that the instrumentation and/or the calibration and analysis algorithms applied to the data could introduce in the interferometric observations. A deep study of all our analysis tools and their effects on noisy data is essential for discerning the reliability of detections of very faint sources. Some discoveries made by pushing the interferometric instruments to their sensitivity limits could turn out to be the result of artifacts produced by the analysis tools.

The main limitations of the phase self-calibration algorithm have been analyzed in many publications (e.g., Linfield 1986; Wilkinson et al. 1988). It is well known that an unwise use of selfcal can lead to imperfect images, even to the generation of spurious source components, elimination of real components, and deformation of the structure of extended sources. In this paper, we focus on the effects that phase self-calibration produces when applied to pure noise. We show that selfcal can generate a spurious source from pure noise, with a relatively high flux density compared to the rms of the visibility amplitudes. We analytically and numerically study how the recoverable flux density of such a spurious source depends on the details of the observations (the sensitivity of the interferometer, the number of antennas, and the averaging time of the selfcal solutions). Finally, we study the effects of phase self-calibration applied to the visibilities resulting from observations of real faint sources, instead of pure noise. We present two simple tests that can be applied to real data in order to check whether a given faint source is real or not, and apply these tests to real data, corresponding to VLBI observations of the radio supernova SN 2004et (Martí-Vidal et al. 2007).

\section{Basics of phase self-calibration}

The basics of phase self-calibration can be found in many publications (e.g., Readhead \& Wilkinson 1978; Schwab 1980). Here, we explain the essence of this algorithm in a few lines. Let us suppose that we have made an interferometric observation using a set of $N$ antennas. We obtain one visibility, $V_{i j}$, for each baseline, that is, for each pair of antennas $(i, j)$. Let us call $\phi_{i j}$ the phase of the visibility $V_{i j}$. Any atmospheric or instrumental effect on the optical path of the signals that arrived to antennas $i$ and $j$ will affect the phase $\phi_{i j}$ in the way:

$\phi_{i j}=\phi_{i j}^{\mathrm{str}}+\phi_{i}^{\mathrm{atm}}-\phi_{j}^{\mathrm{atm}}$ 
where $\phi_{l}^{\mathrm{atm}}$ represents all the undesired (i.e., atmospheric and instrumental) contributions to the optical path of the signal received by the antenna $l$ and $\phi_{i j}^{\text {str }}$ the contribution to the phase that comes purely from the structure of the observed source, that is, the so called source structure phase. It can be easily shown that the quantities known as closure phases, and defined as (Jennison 1958; Rogers et al. 1974):

$C_{i j k}=\phi_{i j}+\phi_{i k}-\phi_{j k}$

are independent of $\phi_{l}^{\mathrm{atm}}$. That is, the closure phases $C_{i j k}$ are only defined by the structure of the observed source. Thus, they are independent of any atmospheric or instrumental contribution that may affect the signals received by the antennas of the interferometer. The phase self-calibration algorithm takes advantage of the closure phases to estimate the undesired antenna-dependent contributions $\phi_{l}^{\text {atm }}$. In short, the phase selfcal finds which set of antenna-dependent quantities $\phi_{l}^{\text {gain }}$ (called phase gains) generate the set of phases:

$\phi_{i j}^{\text {self }}=\phi_{i j}-\phi_{i}^{\text {gain }}+\phi_{j}^{\text {gain }}$,

where $\phi_{i j}^{\text {self }}$ are the phases that better represent the source structure given by the closure phases $C_{i j k}$. In the ideal case, $\phi_{l}^{\text {gain }}=$ $\phi_{l}^{\text {atm }}$ and $\phi_{i j}^{\text {self }}=\phi_{i j}^{\text {str }}$. The process from which the values $\phi_{l}^{\text {gain }}$ (for $l=i, j$ ) are obtained is called hybrid mapping, and its explanation can be found in many publications (e.g., Cornwell \& Wilkinson 1981). Here, suffice to say that the phase gains of the antennas are obtained from a least-square fit of the raw visibilities to the source model obtained from the mapping.

The hybrid mapping is an iterative process from which the structure of the source model is refined step by step. Often, the model used in the first iteration of hybrid mapping is a point source located at the center of the map (obviously, the flux density of this point source will not affect the phase calibration). The successive steps of hybrid mapping and selfcal correct this point source model until the structure that better represents all the closure phases is obtained.

\section{Probability distribution of the visibilities due to pure noise}

When an interferometer observes a given source with a flux density well below the sensitivity limit of its baselines (that is, when the interferometric data contain only noise), both the real and imaginary parts of the resulting visibilities follow Gaussian distributions, centered at the origin. The amplitudes and phases of the visibilities follow distributions different from Gaussian. It can be shown that, for each baseline, the probability distribution of the phases is uniform between $-\pi$ and $\pi$, and that instead the distribution of the amplitudes is given by:

$g(A)=\frac{A}{\sigma_{i j}^{2}} \exp \left(-\frac{A^{2}}{2 \sigma_{i j}^{2}}\right)$

where $g(A)$ is the probability density of the amplitudes and $\sigma_{i j}$ is the width of the Gaussian distributions of the real and imaginary parts of the visibilities of the baseline $(i, j)$. The width $\sigma_{i j}$ is related to the thermal noise of the baseline $(i, j)$. We assume, for simplicity, that all the baselines of the interferometer have the same value of $\sigma$. It can be shown that the rms of the visibility amplitudes of a pure noise signal is $\rho=\sqrt{2} \sigma$ and that the mean amplitude, $\langle A\rangle$, is $\sqrt{\pi / 2} \sigma$, different from zero.

\section{Probability of generating a spurious source from pure noise}

Given that the real part of a visibility with phase in the range $(-\pi / 2, \pi / 2)$ is positive, all the visibilities with phases in that range bring a positive mean flux density to the map. We call phase close to zero to a phase in the range $(-\pi / 2, \pi / 2)$ and phase distant from zero to a phase outside that range.

The distribution of closure phases is uniform between $-\pi$ and $\pi$, as it is also the case for the distribution of phases. This means that there is a subset of closure phases that by chance are close to zero, being compatible with a point source. However, there are also closure phases distant from zero, which are totally incompatible with a compact source. If self-calibration is not applied to the data, then the uniform distribution of phases (and closure phases) will result in a noisy map with no source defined in it. But if a single iteration of phase self-calibration is applied, there is a selection process of the closure phases in the calibration, which may generate a spurious source with a flux density comparable to the rms of the amplitudes (which can be much higher than the rms of the image), as we show below.

For each scan, the effects of the least-square fit described in Sect. 2 can be understood in the following way: selfcal searches for the visibilities corresponding to the antennas most commonly appearing in the closure phases that are close to zero (which usually correspond to phases that can be modelled with a point source). Then, selfcal minimizes the phases of such visibilities by calibrating those antennas, leaving all the other visibilities with the phases dispersed between $-\pi$ and $\pi$. In other words, the phases of the visibilities with large closure phases contribute to increase the value of the $\chi^{2}$ at the minimum, but the position of such minimum only depends on the visibilities with phases that can be modelled with a point source. That is, all the visibilities susceptible of producing a compact source are calibrated and their phases concentrate around zero. All the other visibilities tend to have their phases uniformly distributed between $-\pi$ and $\pi$, thus generating, after the Fourier inversion, a null mean flux density in the map. Thus, selfcal always produces a positive bias in the mean flux density of the map, given that selfcal only acts, effectively, on the phases that can be approached to zero, because their corresponding closure phases are close to zero.

One might think that it is very difficult for an antenna to be involved in a large number of closure phases close to zero, given that the distribution of phases is uniform. One might think that, in average, a given antenna is involved in the same number of closure phases that are close to zero than in the closure phases that are distant from zero. In such case, it would be impossible for selfcal to select which antenna should be calibrated, given that all the antennas participating in each scan would have the same chances for being calibrated. But this is only true in average. In the distributions of all the interferometric observations, there are statistical fluctuations, which are always used by selfcal for the generation of a spurious point source. For a given antenna $i$, the probability of finding $n$ closure phases (in which that antenna is involved) close to zero is:

$P_{i}(n)=\frac{1}{2^{N^{\prime}}} \frac{\left(N^{\prime}\right) !}{n !\left(N^{\prime}-n\right) !}$

where $N^{\prime}=(N-1)(N-2) / 2$ is the total number of closure phases in which antenna $i$ is involed. (Notice that even though all closure phases are not independent, the closure phases with one common antenna are.) Thus, there is a finite probability of finding an antenna that appears in more than $N^{\prime} / 2$ closure phases close to zero. In such cases, the phases of the baselines in which 
the antenna $i$ appears will be minimized with success, generating a positive mean flux density in the map. Actually, even the cases in which $n<N^{\prime} / 2$ can also be used by selfcal for the generation of a spurious point source. In such cases, the closure phases in which antenna $i$ appears will tend to gather around $-\pi$ and $\pi$, meaning that there are other antennas that will appear in a large number of closure phases close to zero (i.e., the antennas belonging to the closure phases distant from zero in which antenna $i$ appears).

In short, there are always statistical fluctuations in the pure noise distribution of phases that can be used by selfcal to produce (by a selection process of the antennas most commonly being involved in the closure phases close to zero) a point source with a spurious source flux density.

It must be said that Global Fringe Fitting, when applied to noisy data, can also generate a spurious source from pure noise, given that this algorithm also finds antenna-based calibrations for adjusting the interferometric fringes of all the baselines in each scan. The spurious source is generated as long as the minimum SNR of the fringes to be considered in the fit is set to a small value (lower than 2 or 3$)^{1}$. In those cases, the Fringe Fitting would work on correlation peaks (fringes) produced, in many cases, by spurious noise fluctuations. Then, by the same reasons given above, there would be a relatively high probability of generating a spurious point source from pure noise.

\section{Dependence of the flux density of the spurious source on the characteristics of the observations}

In this section we consider how the flux density of the spurious source generated by selfcal depends on the parameters defining a set of observations. The parameters that we consider are the sensitivity of the array (for simplicity, we assume the same sensitivity for all the baselines of the interferometric array), the number of antennas of the interferometer, and the averaging time of the selfcal solutions. For the case of the Global Fringe Fitting algorithm, the averaging time of the solutions is equal to the duration of the scans.

\subsection{Numerical study}

We generated a set of synthetic interferometric data with the program FAKE of the Caltech Package (Pearson 1991). We generated $6 \mathrm{~h}$ of observations using a set of 20 antennas. All these antennas had the same diameters $(25 \mathrm{~m})$ and the same system temperatures $(60 \mathrm{~K})$. The correlator integration time was set to $2 \mathrm{~s}$. The source model used by FAKE consisted on a single point source with a flux density of $1 \mathrm{nJy}$ (of course, completely undetectable by the interferometer). The data generated this way thus contain $6 \mathrm{~h}$ of pure noise observations made at 20 identical antennas under identical conditions. The mean of all the visibility amplitudes is $106 \mathrm{mJy}$.

We used the program DIFMAP (Shepherd et al. 1995) for hybrid mapping. We applied the natural weighting scheme to the visibilities, for sensitivity optimization, and applied an initial selfcal using a centered point source. The hybrid mapping steps were repeated until the $\chi^{2}$ of the fit of selfcal arrived to convergence. Then, we deconvolved the resulting point source using

\footnotetext{
${ }^{1}$ Even though a SNR of $2-3$ is not generally used in the calibration of typical observations (being SNR cutoffs of $\sim 5$ more common), low SNR cutoffs may be applied with very small delay/rate windows, say, after a phase-reference pre-calibration.
}

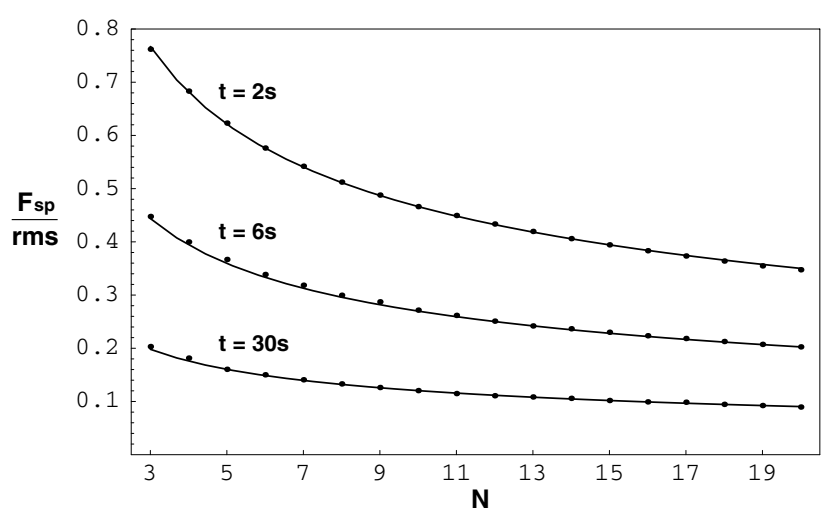

Fig. 1. Flux densities of an spurious point source, $F_{\mathrm{sp}}$, in units of amplitude rms, $\rho$, recovered from hybrid mapping using pure noise synthetic visibilities, as a function of the number of antennas $(N)$ and the averaging time of the selfcal solutions $(t)$. The lines correspond to our model (Eq. (7)) and the dots to the numerical simulations.

the CLEAN algorithm to see how much flux density was generated by selfcal. This process was repeated for different numbers of antennas and for different averaging times of the selfcal solutions. The spurious point source flux densities obtained in all these cases are shown in Fig. 1 as filled circles.

\subsection{Analytical study}

When the interferometer observes only noise, the sensitivity of the baselines defines the value of the standard deviation, $\sigma$, of the Gaussian distributions of the real and imaginary parts of the visibilities. As the sensitivity increases, the thermal noise of the baselines decreases, decreasing also the value of $\sigma$. Given that selfcal has to do only with phases and leaves the amplitudes unaltered, the recoverable flux density of the spurious source depends linearly on the rms of the amplitudes, which in turn also depends linearly on the value of $\sigma$. Thus, an increase in the sensitivity of the interferometer decreases the amount of spurious flux density recoverable from the data using selfcal. The constant factor in the ratio between the flux density recovered and the rms of the visibility amplitudes depends on the method used to estimate the flux density (i.e. different deconvolution algorithms or modelfitting to the visibilities). In our case, from the numerical study described in the previous section, we determine it to be $0.907 \pm 0.002$.

The averaging time of the selfcal solutions also has an effect on the spurious source flux density. If we have one observation every $t_{0}$ seconds (usually, $t_{0}$ is $2 \mathrm{~s}$ ) and find only one solution of selfcal every $t$ seconds, with $t>t_{0}$, then the spurious source flux density decreases. Finding a single solution of selfcal every $t$ seconds is equivalent to averaging all the observations in bins of $t$ seconds and, afterwards, self-calibrating the resulting visibilities. When we average the visibilities in blocks of $t$ seconds, we are averaging separately the real and imaginary parts of the visibilities, which follow Gaussian distributions. The effect of this average is that the standard deviations of the resulting distributions decrease by a factor $\sqrt{t / t_{0}}$, because of the Central Limit Theorem.

The dependence of the spurious source flux density on the number of antennas is more difficult to find. There are lots of possible combinations of phases and baselines that help selfcal to generate a spurious source, and each one of these combinations has a different weight in the final spurious flux density. We can use a simplified phenomenological model to find out the 
recovered flux density as a function of the number of antennas. In principle, the recovered flux density depends directly on how well the point model fits the data. A good indicator of the $\mathrm{ad}$ justability of the data, with the phases randomly distributed, is, for each scan, equal to the number of visibility phases divided by the number of phase gains to fit. That is, the number of phases per parameter. When the number of phases per parameter increases, one single parameter must account for the minimization of more phases, and the quality of the fit decreases. In our case, the number of parameters is equal to $N-1$, given that one antenna (the reference antenna) has a null phase gain by definition. Thus, the number of phases per gain to be fitted is:

$\frac{\# \text { phases }}{\# \text { gains }}=\frac{N(N-1)}{2} \frac{1}{N-1}=\frac{N}{2}$.

We can now look for a model of the dependence of the recovered spurious source flux density on the number of antennas using the quantity $N / 2$ as variable. From the numerical simulations described in the previous section, we have found that the spurious source flux density is fitted very well using the simple model $F_{\text {sp }} \propto(N / 2)^{\gamma}$, with $\gamma=-0.413 \pm 0.001$.

Taking these considerations into account, the recoverable flux density using selfcal on a set of randomly distributed data can be written as:

$F_{\mathrm{sp}}=0.907 \rho \sqrt{\frac{t_{0}}{t}}\left(\frac{N}{2}\right)^{-0.413}$

where $F_{\text {sp }}$ is the spurious source flux density that can be generated by selfcal, $\rho$ is the root-mean-square (rms) of the visibility amplitudes, $t_{0}$ is the averaging time used in the correlator (typically, $\left.t_{0}=2 \mathrm{~s}\right), t$ is the averaging time of the selfcal solutions, and $N$ is the number of antennas of the interferometer. We note that the duration of the whole set of observations does not affect $F_{\mathrm{sp}}$, since this flux density depends on the ratio between the number of phases close to zero and the number of phases distant from zero, but does not depend on the total amount of visibilities used in the Fourier inversion. This model is shown in Fig. 1.

\subsection{The use of selfcal in specific situations}

Equation (7) gives an estimate of the contribution of the artifacts of selfcal to the flux density of a source obtained by calibrating the antennas with the hybrid mapping technique. For cases of high SNR data, such contribution to the total flux density of the sources is negligibly small. However, when the flux density of a source is comparable to the rms of the visibility amplitudes, care must be exercised with the use of selfcal or the Global Fringe Fitting algorithm.

We note that in the worst situation for the use of selfcal (i.e., 3 antennas and $t=t_{0}$ ) the amount of spurious source flux density is as large as $76 \%$ of the rms of the visibility amplitudes. For 10 antennas (the case of the VLBA) the recoverable flux density decreases to $46 \%$ of the rms (and can be lower if we set $t>t_{0}$ ). For interferometers with a large number of antennas, the amount of spurious source flux density is, of course, smaller. For example, if we extrapolate the results shown in Fig. 1 to 50 antennas (the case of ALMA), the spurious source flux density generated by selfcal would be $24 \%$ of the rms of the visibility amplitudes, using $t=t_{0}$.

All these results assume the same sensitivity for all the baselines. In real cases, each baseline has its own sensitivity, with the longest baselines noisier than the shortest ones. The use of data from all the baselines in the fit can worsen the situation. A good alternative for avoiding the spurious source generated by selfcal or, at least, to make its flux density smaller is to flag or downweight the longest baselines in the fit and/or to increase the statistical weight of the data coming from the most sensitive antennas of the array. Nevertheless, even doing so, the statistical fluctuations of the closure phases will always tend to make, after the use of selfcal, a spurious source with a considerably large flux density.

A better way to calibrate faint source data is using the phasereference technique (e.g., Beasley \& Conway 1995). When using this technique, scans of a strong (reference) source are introduced between the scans of the faint (target) source. Then, the antenna gains are determined from the observations of the strong source and then interpolated to the scans of the faint (target) source. This technique is rather insensitive to the artifacts of selfcal and the probability of generating a fake signal from noise is practically zero. This is so, because the calibration of the target source comes from the analysis of data coming from another source (the reference source). Therefore, the noise in the data of the faint source does not affect the antenna calibrations. However, it is common to use the antenna gains determined from the phase reference as an a priori calibration, performing then a Global Fringe Fitting on the target source data using small search windows (based on the calibration from the reference source data) or applying self-calibration to the target visibilities in order to improve the dynamic range of the final image. In some cases, this might be malpractice, because the probability of generating a spurious source flux density from noise appears anew with full strengh, wasting all the benefits of the phase referencing.

\section{Tests of the reliability of a source detection from noisy data}

In this section we present two simple tests that can be performed on real data in order to check the reliability of a possible source detection, or to check if part of the flux density of a detected source may come from artifacts of selfcal. These tests are only meaningful when they are applied to extremely noisy data. However, the application of these tests to high SNR data can still give us an idea of the possible contribution of the artifacts of selfcal to the flux density recovered from a source.

We assume, in all our discussion, that the detected source is compact enough to be considered point-like, without any important loss of precision. As in the previous sections, to simplify the expressions we also assume that all the baselines of the interferometer have the same sensitivity.

\subsection{Test based on the averaging time of the selfcal solutions}

The dependence of the spurious source flux density on the averaging time of the selfcal solutions is determined by the dependence of the standard deviation $\sigma$ on the averaging time. That dependence translates into Eq. (7). However, the flux density of a real source in the data is independent of the averaging time of the selfcal solutions. We can use this condition to estimate the flux density of a (possibly real) source detected under critical circumstances. If we apply phase self-calibrations to a real data set for different averaging times, $t$, of the selfcal solutions, the flux densities recovered after each self-calibration, $F_{\text {self }}$, are given by the formula:

$F_{\text {self }}=F_{\text {sp }}+F_{\text {real }}=\frac{K}{\sqrt{t}}+F_{\text {real }}$ 


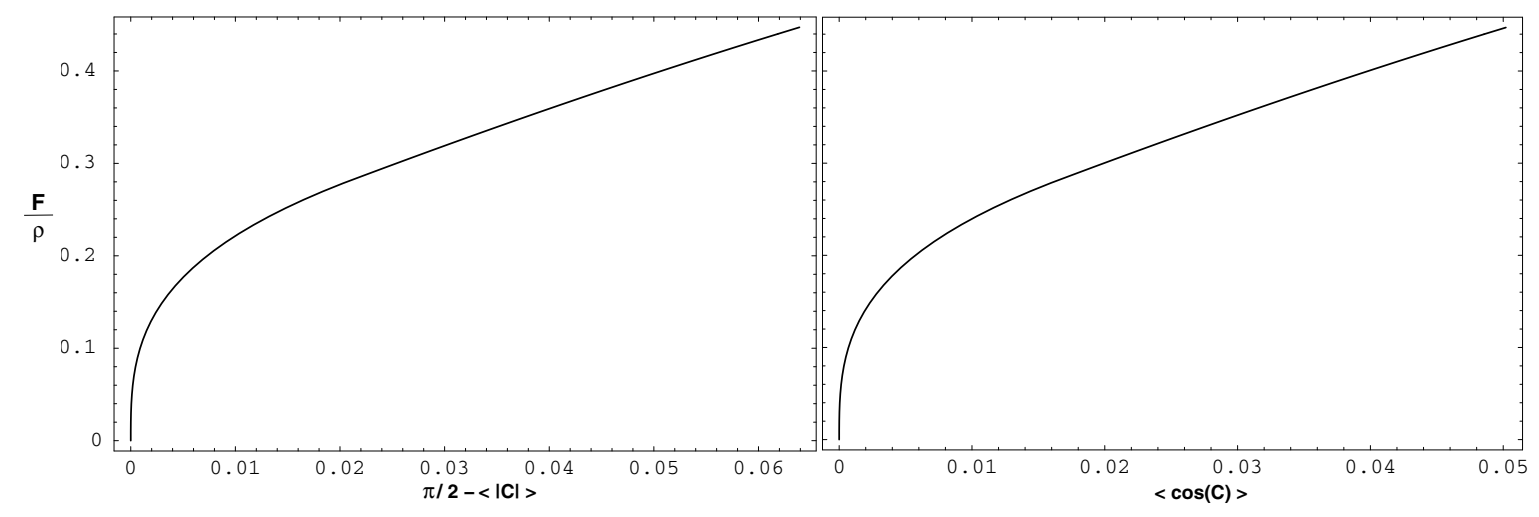

Fig. 2. Theoretical dependence of the flux density of a real observed source (in units of the rms of the visibility amplitudes), as a function of " $\pi / 2$ minus the mean absolute values of the closure phases" (left side) and as a function of the mean cosine of the closure phases (right side). These theoretical behaviors have been obtained from Eq. (10).

where $K$ (related to the specifics of the interferometer) and $F_{\text {real }}$ (an estimate of the flux density of the real source) are parameters to be fitted.

\subsection{Test based on the distribution of closure phases}

In the case that the signal of a real source is included in the visibilities, the probability distributions of the real and imaginary parts are still Gaussian, but the mean value of the real part of the visibilities (if such visibilities are well calibrated) will be equal to the flux density of the real source. Then, it can be easily shown that the probability distribution of the resulting visibility amplitudes and phases is:

$g(A, \phi)=\frac{A}{\sigma^{2}} \exp \left(-\frac{(A+F)^{2}}{2 \sigma^{2}}\right) \exp \left(\frac{A F \cos \phi}{\sigma^{2}}\right)$

where $g(A, \phi)$ is the probability density of amplitudes (variable $A$ ) and phases (variable $\phi), F$ is the flux density of the real source, and $\sigma$ is the width of the distributions of the real and imaginary parts of the visibilities.

Equation (9) turns into Eq. (4) for $F=0$. When $F$ is different from zero, the distribution of phases is not uniform. As $F$ increases, the phases gather around zero in a Gaussian-like manner. The distribution of amplitudes also changes, increasing the ratio between the rms of the visibility amplitudes and $\sigma$.

How could the information provided by Eq. (9) be used to check the reliability of a possible source detection? The closure phases are robust quantities that can be used to check the reliability of a source detection. The closure phases are sensitive to $F$ and are not affected by the phase self-calibration. The distribution of closure phases tends to gather around zero if there is signal of a real source in the data (and particularly so if the source has no structure) and is uniformly distributed if there is only noise in the data. From the definition of closure phase (see Eq. (2)) we conclude that, if the visibility phases are well calibrated, the probability distribution of closure phases is equal to:

$c(\beta)=\int_{-\pi}^{\pi} \int_{-\pi}^{\pi} p\left(\phi_{1}\right) p\left(\phi_{2}\right) p\left(\beta-\phi_{1}-\phi_{2}\right) \mathrm{d} \phi_{1} \mathrm{~d} \phi_{2}$

where $c(\beta)$ is the probability density of the closure phase, $\beta$, and

$p(\phi)=\int_{0}^{\infty} g(A, \phi) \mathrm{d} A$.

Let us ellaborate on this: for the case of a perfect calibration, the phases of data from a given baseline $(i, j)$ are independent of the phases from any other baseline, given that all the contributions to $\phi_{i j}$ come from noise, which is uncorrelated between the different baselines. Thus, the probability distribution function of any linear combination of visibility phases (as it is the case of the closure phase) is equal to the product of the probability distributions of the visibility phases. However, for the case of a non-perfect calibration, in which selfcal has introduced a spurious source flux density in the data, the distributions of the phases from the different baselines will no longer be independent and Eq. (10) will not quite apply. In such cases, the phase distributions, $p(\phi)$, will be more peaked around zero, but correlations will appear among the phases of the different baselines. Therefore, the probability distribution function of a linear combination of phases will not be equal to a simple product of $p(\phi)$. Nonetheless, the correlations between baselines that selfcal introduces in the data keep the distribution of closure phases, $c(\beta)$, unaltered. That is, even after a selfcal iteration has generated a spurious source in the map, the distribution of closure phases is still equal to $c(\beta)$, as computed from the distribution of the phases, $p(\phi)$, corresponding to perfectly calibrated data.

This invariance property of the closure phase distribution, $c(\beta)$, can be used to check the reliability of a possible source detection. In Fig. 2, the theoretical flux density of a real source (in units of the rms of the amplitudes, $\rho$ ) is shown as a function of the mean absolute value of the closure phases and as a function of the mean cosine of the closure phases. Both quantities are directly related to the deviation of the closure phase distribution from a uniform distribution. If the closure phases are uniformly distributed between $-\pi$ and $\pi$, the average of their absolute values will be equal to $\pi / 2$ and the mean cosine will be equal to zero. Any concentration of closure phases around zero (i.e., the existence of any real source in the data) will result in a decrease of the first value and an increase of the second.

If a faint source is detected and the reliability of the detection needs to be checked, the average of the absolute values of the closure phases of the observations (or the average of their cosines) can be computed and compared in Fig. 2 to determine how concentrated around zero the closure phases are. This way, it can be seen whether the flux density of our detection is consistent with a real source or not.

\subsubsection{Comments on the closure-phases test}

At this stage, it must be said that, even though the self-calibration does not affect the closure phases, a time average of a set of self-calibrated visibilities will change the closure phase 
distribution. That is, if selfcal generates a spurious source from noise and the visibilities are averaged in time bins of $t$ seconds, with $t>t_{0}$, the closure phase distribution changes and the resulting closure phases concentrate around zero, creating the effect of a source that is completely indistinguishable from a real one using any test.

For very small values of the flux density $F$, this test is not as good as the first one. As we can see in Fig. 2, the closure phases do not dramatically change their distribution for flux densities in the range between 0 and $\sim 15 \%$ of the rms of the visibility amplitudes. For tentative detections under critical circumstances in that range, this test could lead us to the wrong conclusion about the reliability of a source detection. Looking at it from a different viewpoint, Fig. 2 provides an interesting lesson: for a given dataset, there can exist a real faint source (appearing in a map with a dynamic range of 6 or more) even if the distribution of closure phases is uniform, that is, even if the closure phase distribution is noise-like. Thus, a conclusion on the reliability of a source detection based only on the closure phase distribution being extremely noisy, is not definitive.

Another thing worth-noticing is that this test assumes the same sensitivity for all the antennas and a source compact enough for generating closure phases close to zero even for the longest baselines (which may not be the case, specially for sources with a very low flux density per unit beam). These assumptions impose limitations to the use of this test. However, it could still be applied by restricting its use to the shortest baselines with similar antennas. For an array with a large antenna, the closure phases in which that antenna appears could still be used (for sensitivity optimization in the closure phase distribution), but then the flux density estimated from the amplitude rms might have a bias produced by the very different rms in different baselines.

We must also note that in cases of high SNR, the rms of the visibility amplitudes is no longer related only to the thermal noise of the baselines (the flux density of the source affects the value of the rms), and the fraction $F / \rho$ shown in Fig. 2 should be accordingly corrected. For cases of high SNR, the quantity that will substitute $\rho$ in the fraction $F / \rho$ shown in Fig. 2 is $\sqrt{\rho^{2}-F^{2}}$.

\subsection{Application to real cases}

Real data do not obey the simplifying assumptions that we have used in the earlier sections. The baselines of a real interferometer have different sensitivities, which also vary in time. Thus, in order to check the reliability of a source detection from real data we must search a subset of observations in which the sensitivity of the antennas is approximately constant. Moreover, we must only work with the subset of most sensitive antennas of the interferometer. If there is one antenna in our interferometer that is clearly more sensitive than the others, we should compute only the average of the closure phases in which this more sensitive antenna appears, in order to insure the possible signature of the source in the closure phase distribution. In what follows, we apply our reliability criteria to real data corresponding to the radio supernova SN 2004et (Martí-Vidal et al. 2007).

\subsubsection{Supernova SN2004et}

We observed this supernova on 20 February 2005. From all data reported in Martí-Vidal et al. (2007), we have chosen the following subset of antennas: Brewster, Fort Davis, Green Bank, Hancock, Kitt Peak, and Owens Valley. We have only computed the closure phases in which the antenna Green Bank appears, and we have used data only from $14 \mathrm{~h}$ to $20 \mathrm{~h}$ (UT). These choices are based on the quality of the data for our purposes (i.e., the stability of the antenna sensitivities, which we assume proportional to the system temperatures registered for each station).

First test: we self-calibrated the SN 2004et data using different averaging times, ranging from 2 to $120 \mathrm{~s}$ (roughly, the duration of one scan). The fit of the flux densities recovered from the SN 2004et data as a function of the averaging time of the selfcal solutions, Eq. (8), results in a value of $F_{\text {real }}=0.90 \pm 0.13 \mathrm{mJy}$. This value is clearly higher than zero, indicating that there is a real signal in the data. This value is also close to the flux density of SN 2004et reported by Martí-Vidal et al., $0.87 \pm 0.03 \mathrm{mJy}$, recovered from phase referenced data with a deconvolution using CLEAN.

Second test: even though we know that it is not quite appropriate, we have also performed this test on the SN 2004et data. The flux density of SN2004et is too low compared to the rms of the visibility amplitudes $(\sim 20 \mathrm{mJy})$ for obtaining a good result with the test of the closure phase distribution. The average value of the cosines of all the closure phases considered in these observations is equal to $0.005 \pm 0.002$. This average value is slightly higher than 0 . The average of the absolute values of the closure phases is $0.0065 \pm 0.0020$. From Fig. 2 we estimate a flux density of the supernova of $0.18 \pm 0.08$ times the rms of the visibility amplitudes, $\rho$, used in our computations, which, as said above, is $\sim 20 \mathrm{mJy}$. Hence, the estimated flux density of the (real) source in the data is, then, $3.8 \pm 1.6 \mathrm{mJy}$. This value is too high, but compatible (at a 2-sigma level) with the flux density estimated from the other reliability test. As expected, also the uncertainty of the flux density estimated is much higher in this case.

Thus, the reliability tests for this source are successful. We must note that Martí-Vidal et al. calibrated the data of SN 2004et using the phase-reference technique, in which they interpolated the antenna gains obtained from the observations of the source $\mathrm{J} 2022+614$ to the scans of the supernova. These authors did not refine afterwards such a calibration applying a Global Fringe Fitting to the supernova data. This procedure assured a reliable detection of the supernova. These authors did not apply any other calibration (selfcal) to the phased-referenced supernova data, in order to avoid any possible artifact introduced by the use of selfcal.

\section{Conclusions}

We have analyzed the consequence of the phase-self-calibration algorithm when it is applied to extremely noisy data. We have studied how this algorithm and the statistical fluctuations of the visibility phases can create a spurious source from pure noise. The flux density of the spurious source can be a considerable fraction of the rms of the visibility amplitudes. The application of other other antenna-based calibration algorithms (like the Global Fringe Fitting) to noisy data can have similar consequences to those of selfcal if the SNR cutoff of the gain solutions is set to small values.

We have considered numerical and analytic studies to show how the flux density of a spurious source created by selfcal depends on the number of antennas, the sensitivity of the array, and the averaging time of the selfcal solutions. We have also presented two simple tests that can be applied to real data in order to check if the detection of a faint source could be the result of the application of an antenna-based calibration algorithm to noisy data. These tests basically relate the averaging time of the 
selfcal solutions and the characteristics of the closure phase distribution to the flux density of a compact source possibly present in the data. To show a practical case, we have applied these tests to a set of real VLBI observations of supernova SN 2004et and found good agreement between the flux density recovered by CLEAN from the (phase-referenced) visibilities of this supernova (Martí-Vidal et al. 2007) and the flux density estimate provided by our reliability tests.

Acknowledgements. This work has been partially funded by Grants AYA200422045-E and AYA2005-08561-C03-03 of the Spanish DGICYT. We agree the anonymous referee for his/her very helpful comments, corrections, and suggestions.

\section{References}

Beasley, A. J., \& Conway, J. E. 1995, Very Long Baseline Interferometry and the VLBA, ASP Conf. Ser., 82, 328

Cornwell, T. J., \& Wilkinson, P. N. 1981, MNRAS, 196, 1067

Cotton, W. D. 1979, AJ, 84, 1122

Jennison, R. C. 1958, MNRAS, 118, 276

Linfield, R. P. 1986, AJ, 92, 213

Martí-Vidal, I., Marcaide, J. M., Alberdi, A., et al. 2007, A\&A, 470, 1071

Pearson, T. J. 1991, BAAS, 23, 991

Readhead, A. C. S., \& Wilkinson, P. N. 1978, ApJ, 223, 25

Rogers, A. E. E., Hinteregger, H. F., Whitney, A. R., et al. 1974, ApJ, 193, 293

Shepherd, M. C., Pearson, T. J., \& Taylor, G. B. 1995, BAAS, 26, 987

Schwab, F. R. 1980, Proc. Soc. Photo-Opt. Instrum. Eng., 231, 18

Schwab, F. R., \& Cotton, W. D. 1983, AJ, 88, 688

Wilkinson, P. N., Conway, J., \& Biretta, J. 1988, IAUS, 129, 509 